\title{
Restoring Mesh Independency in FEM-DEM Multi-scale Modelling of Strain Localization Using Second Gradient Regularization
}

\author{
J. Desrues, A. Argilaga, S. Dal Pont, G. Combe, D. Caillerie and T. kein \\ Nguyen
}

\begin{abstract}
Continuum media from classical mechanics cannot appropriately reproduce the evolution of materials exhibiting strong heterogeneities in the strain field, e.g. strain localization. Models without a microscale representation cannot properly reproduce the microscale mechanisms that trigger the strain localization, in addition, first gradient relations don't present any length parameter in the formulation. This results in a model without a characteristic length that cannot exhibit any objective band width. In this paper, techniques to introduce an internal length will be enumerated. Microstuctured materials will be retained and in particular Second Gradient model will be exposed and used along with a FEMxDEM approach. Numerical results showing the abilities of the enriched model will conclude the text.
\end{abstract}

\section{Introduction}

The idea of FEM $\times$ DEM is to solve a continuum boundary value problem (BVP) at the macroscale while obtaining the constitutive material behaviour from a DEM

\footnotetext{
J. Desrues (四)

CNRS - Université Grenoble Alpes, Grenoble, France

e-mail: jacques.desrues@3sr-grenoble.fr
}

A. Argilaga · S. Dal Pont · G. Combe · D. Caillerie · T. kein Nguyen Université Grenoble Alpes, Grenoble, France

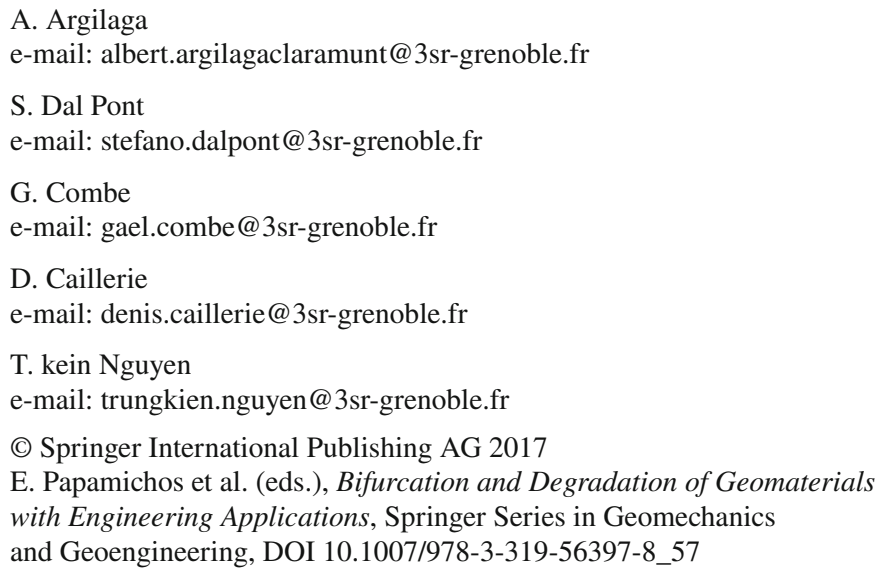


microscale in a fully coupled hierarchical multiscale method. Some early works $[11,16,20]$, have put in evidence the potential of the method to provide a refined description of complex constitutive behaviors. Indeed, FEM $\times$ DEM methods allow to couple the advantages of Discrete Elements and the efficiency of Finite Elements. Later works have enhanced and extended this approach to the study of anisotropy [7, 18], granular cohesion [19], material heterogeneity [25], real scale engineering applications [8, 18], more realistic constitutive behaviors using 3D DEM [12, 26], macroscale hydro-mechanical coupling [10, 26]. More recently, [12] have embedded non-local regularization at the macro-scale and [9] has developed a full micro-macro $3 \mathrm{D}$ approach.

It is known that for a strain softening material the initiation of strain localization can lead to an ill-posed Partial Differential Equation (PDE) problem [23]. The consequence of this ill posedness are numerical instabilities and strain mesh dependency [22]. Regularization techniques have been developed to overcome these problem; both nonlocal [5] and local [6, 17] approaches exist. An example of nonlocal regularization in a FEM $\times$ DEM model is presented in [12]. Local formulations use a local relationship between stress and strains in the same manner as classical constitutive relations are defined. Second gradient model, as a particular case of the Germain theory [6] has been developed [1, 4, 14, 21]. It has been extensively applied in geomechanics and engineering applications with satisfactory results [3, 13, 24].

Previous developments of FEM $\times$ DEM could not take advantage of Second Gradient regularization due to the poor solution stability and limited available mesh refinement. Recent improvements concerning stability and computational efficiency allow to build a FEM $\times$ DEM model including local Second Gradient. This results in an objective model capable of simulating real scale problems with any mesh refinement.

\section{Introduction of an Internal Length}

Models using a first-order constitutive relation of classical mechanical continuum cannot properly predict the behaviour of a medium with high strain gradients. Those approaches suffer from non-objectivity due to mesh dependency in localization problems.

Possible causes: the first-order constitutive relation does not give any information about the internal length of the model; due to that, the localization band thickness will tend to shrink to a size proportional to the mesh size. In this way, if the mesh is refined making the size of elements tend to zero in order to get an exact solution, the strain will concentrate in a null size band posing obvious problems.

It's needed to set a relationship between the micro-scale heterogeneity and the macro-scale characteristic length in order to establish a proper micro-scale size effect on the macro-scale. An enriched model with microstructure is proposed: local second gradient model [1, 4, 14, 21]. 

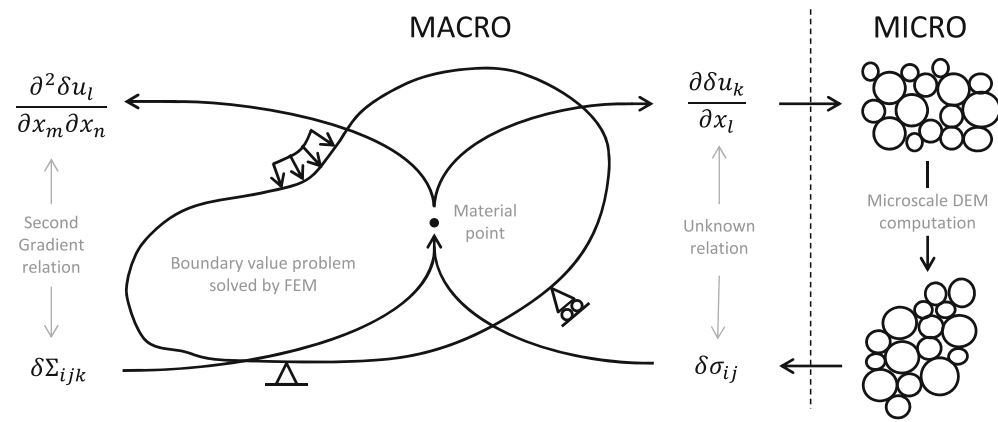

Fig. 1 Computational homogenization scheme with Second Gradient

\section{Local Second Gradient Model}

Microstructured material descriptions consider a continuum field in the micro-scale enriched by higher order terms [6]. In this way, the kinematics of the media is enriched by its micro-scale introducing a local dependence on an internal length parameter. This characteristic length [1] regularizes the solution making strain localization mesh independent. A local formulation complies with the principle of local action so it states a stress-strain relationship in the same manner as a classical constitutive laws do, this makes the implementation of local regularization in classical models a straight forward procedure (Fig. 1).

We present the weak form of the balance equations written for the strain gradient theory viewed as a particular case of the microstructured continuum theory [2]:

$$
\int_{\Omega^{t}}\left(\sigma_{i j}^{t} \frac{\partial u_{i}^{\star}}{\partial x_{j}^{t}}+\sum_{i j k}^{t} \frac{\partial^{2} u_{i}^{\star}}{\partial x_{j}^{t} \partial} x_{k}^{t}\right) d \Omega^{t}-\bar{P}_{e}^{\star}=0
$$

where, superscripts $t$ and $\star$ denote, quantities at a given time $t$ and virtual quantities, $\sigma_{i j}^{t}$ is the Cauchy stress tensor, $\Sigma_{i j k}^{t}$ is the corresponding double stress tensor, $u_{i}^{\star}$ is a kinematically admissible virtual displacement feld, $x_{i}^{t}$ are the current coordinates of the points of the studied body and $P_{e}^{\star}$ is the external virtual work generated by the corresponding external forces [15].

\section{Numerical Simulations}

A compression biaxial test with second gradient enrichment shows how the regularization turns the shear band patterns independent from the mesh size (Fig. 2). This regularization not only enriches the physics of the model but also accelerates the simulation because of an improvement of the iterative efficiency, i.e. a regularized 

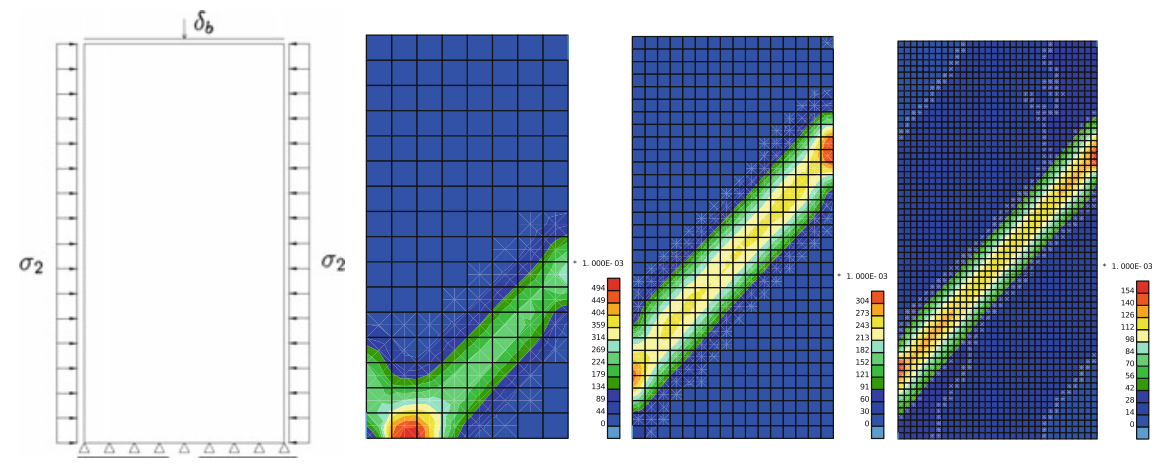

Fig. 2 Biaxial compression test boundary condition. Results with different mesh size: 128, 512 and 2048 elements. Band width independent of mesh size

problem has less possible solutions meaning that the Newton scheme will converge faster.

\section{Conclusion}

A second gradient regularization has been presented. First gradient mechanical models do not present any internal length, this poses some issues as the problem undergoes softening and strain localization, i.e. mesh dependency. Second gradient is used to provide the model with an internal length and to regularize the problem.

Second gradient is a microstructured local model, this means that the relation can be applied in a material point in the same fashion as a classical constitutive law is. The regularization is of special interest in the FEM $\times$ DEM model; because of the noisy behaviour of the DEM constitutive law the problem is very likely to lose its ellipticity leading to an ill posed problem. The second gradient allows to soften the loss of ellipticity not only regularizing the problem but also allowing for a faster convergence.

Results are presented, Compression biaxial test simulations with a second gradient enrichment show that the regularization sets an internal length which makes the model mesh independent as far as the mesh size is fine enough.

\section{References}

1. Chambon, R., Caillerie, D., El Hassan, N.: One-dimensional localisation studied with a second grade model. Eur. J. Mech.-A/Solids 17(4), 637-656 (1998)

2. Chambon, R., Caillerie, D., Matsuchima, T.: Plastic continuum with microstructure, local second gradient theories for geomaterials: localization studies. Int. J. Solids Struct. 38(46), 8503$8527(2001)$ 
3. Collin, F., Chambon, R., Charlier, R.: A finite element method for poro mechanical modelling of geotechnical problems using local second gradient models. Int. J. Numer. Methods Eng. 65(11), 1749-1772 (2006)

4. De Borst, R., Mühlhaus, H.-B.: Gradient-dependent plasticity: formulation and algorithmic aspects. Int. J. Numer. Methods Eng. 35(521-539), 1992 (1992)

5. Eringen, A.C.: Nonlocal polar elastic continua. Int. J. Eng. Sci. 10(1), 1-16 (1972)

6. Germain, P.: La méthode des puissances virtuelles en mécanique des milieux continus. J. Mécanique 12, 236-274 (1973)

7. Guo, N., Zhao, J.: A hierarchical model for cross-scale simulation of granular media. AIP Conf. Proc. 1542, 1222-1225 (2013)

8. Guo, N., Zhao, J.: Multiscale insights into classical geomechanics problems. Int. J. Numer. Anal. Methods Geomech. (2015)

9. Guo, N., Zhao, J.: 3D multiscale modeling of strain localization in granular media. Comput. Geotech. (2016)

10. Guo, N., Zhao, J.: Parallel hierarchical multiscale modelling of hydro-mechanical problems for saturated granular soils. Comput. Methods Appl. Mech. Eng. 305, 37-61 (2016)

11. Kaneko, K., Terada, K., Kyoya, T., Kishino, Y.: Global-local analysis of granular media in quasi-static equilibrium. Int. J. Solids Struct. 40(15), 4043-4069 (2003)

12. Liu, Y., Sun, W., Yuan, Z., Fish, J.: A nonlocal multiscale discrete-continuum model for predicting mechanical behavior of granular materials. Int. J. Numer. Methods Eng. (2015)

13. Marinelli, F.: Comportement couplé des géomatériaux: deus approches de módelisation numérique. Ph.D. thesis (2013)

14. Matsushima, T., Chambon, R., Caillerie, D.: Second gradient models as a particular case of microstructured models: a large strain finite elements analysis. Comptes Rendus de l'Académie des Sciences-Series IIB-Mechanics-Physics-Astronomy 328(2), 179-186 (2000)

15. Matsushima, T., Chambon, R., Caillerie, D.: Large strain finite element analysis of a local second gradient model: application to localization. Int. J. Numer. Methods Eng. 54(4), 499$521(2002)$

16. Miehe, C., Dettmar, J., Zäh, D.: Homogenization and two-scale simulations of granular materials for different microstructural constraints. Int. J. Numer. Methods Eng. 83(8-9), 1206-1236 (2010)

17. Mindlin, R.D.: Second gradient of strain and surface-tension in linear elasticity. Int. J. Solids Struct. 1(4), 417-438 (1965)

18. Nguyen, T.: Modélisation numérique à double échelle des matériaux granulaires cohésifs: Approche par éléments finis-éléments discrets. Ph.D. thesis (2013)

19. Nguyen, T., Combe, G., Caillerie, D., Desrues, J.: FEM $\times$ DEM modelling of cohesive granular materials: numerical homogenisation and multi-scale simulations. Acta Geophysica 62(5), 1109-1126 (2014)

20. Nitka, M., Combe, G., Dascalu, C., Desrues, J.: Two-scale modeling of granular materials: a DEM-FEM approach. Granul. Matter 13(3), 277-281 (2011)

21. Pamin, J.K.: Gradient-dependent Plasticity in Numerical Simulation of Localization Phenomena. Delft University of Technology, TU Delft (1994)

22. Pietruszczak, S., Mroz, Z.: Finite element analysis of deformation of strain-softening materials. Int. J. Numer. Methods Eng. 17(3), 327-334 (1981)

23. Rice, J.R.: The Localization of Plastic Deformation. Brown University, Division of Engineering (1976)

24. Salehnia, F., Collin, F., Li, X.L., Dizier, A., Sillen, X., Charlier, R.: Coupled modeling of excavation damaged zone in boom clay: Strain localization in rock and distribution of contact pressure on the gallerys lining. Comput. Geotech. 69, 396-410 (2015)

25. Shahin, G., Desrues, J., Dal Pont, S., Combe, G., Argilaga, A.: A study of the influence of rev variability in double scale FEM $\times$ DEM analysis. Int. J. Numer. Methods Eng. (2016)

26. Wang, K., Sun, W.: A semi-implicit discrete-continuum coupling method for porous media based on the effective stress principle at finite strain. Comput. Methods Appl. Mech. Eng. 304, 546-583 (2016) 